\title{
An Overview of Cough Sounds Analysis
}

\author{
Chenwei Zhang ${ }^{1, \text { a }}$ \\ ${ }^{1}$ School of Information Engineering, Guangdong University of Technology, Guangzhou 510006, \\ China \\ a Chenwei_Cheung@163.com
}

Keywords: Cough sounds analysis, Assessing, Discrimination, Application

\begin{abstract}
Cough is a common respiratory symptom, caused by the inflammation of trachea and bronchial mucosa or chemical stimulation. Compared with other sounds, cough sound has a typical uniqueness which makes it possible to analyze the differences between cough and other sound performance. Since coughing is a very important symptom of many diseases, the study of the relationship between the nature of coughs and the pathogenesis of respiratory diseases has considerable medical value. Despite the constant progress in cough-study over these years, attention to this interesting physical and clinical problem is still insufficient to fully address various objective problems, including the correct cognition of cough-mechanism and the relationship with various respiratory diseases. This paper summarizes the previous research on cough sounds, including the method of analyzing cough and the difficulties in current cough analysis. The idea and prospect for further research in the future is also mentioned.
\end{abstract}

\section{Introduction}

Cough is one of the most common symptoms of respiratory disease and is also one of the body's defense mechanisms. It prevents some harmful substances from entering the respiratory system and removes potentially harmful debris from the lungs and respiratory tracts [1]. Cough is a very important feature of more than 100 diseases and other medical symptoms. As reflex-generated perturbation of the respiratory function cough is an important symptom in many respiratory diseases or irritations. [2] Therefore, the study of the mechanism of the cough and the analysis of the cough has a considerable clinical importance [3-9]. In clinical diagnosis, accompanied by cough symptoms are usually pertussis, asthma, laryngitis and so on. Thus, the spittoon is potentially an observation of considerable importance, because it is a way in which we can classify and quantify the cough reflex which is one of the commonest symptoms of respiratory diseases [10].

In addition, the study of the mechanism of cough is also very necessary. It is well known that coughing is presented by a sudden expulsion of air which is accompanied by a typical sound. This vocal feature allows it to be identified and to be distinguished from other vocal manifestations [10]. Since cough is a powerful response to the stimulation of sensory nerves predominantly in the larynx, trachea and central airway, the resulting cough reflex mechanism involves both the sub- and supra-glottal region and the cough sound might either be vocal or produced in the airway branching network [2, 10].

To achieve a better understanding of the mechanism of cough sound creation, it is attempted to interpret time and spectral features in relation to the localization of cough sound production. Despite some of cough's properties were already described by ancient physicians 2000 years ago, (e.g. Aulus Cornelius Celsus, Areteus of Cappadocia, etc.) [10] it is really a pity that cough sound utilized as a diagnostic tool is still absent from current medical practice. Therefore, collecting patient's cough sounds and establishing a cough pathology database is of great significance for future medical practice and this field has drawn the attentions of both physiologists and clinicians.

At present, there are two main directions for the analysis of cough sounds. One is the registration of acoustic pressure accompanying coughing in a form of time-amplitude wave. Another method is to analyze the time-frequency of the cough sounds. Both methods are unavoidably faced with the problem of how to collect the cough without distortion. This paper introduced some methods of 
collection and analysis about cough sounds in the past, and put forward the ideas and prospects for the future research and potential application in this field.

\section{Assessing Cough Sounds}

The simple, noninvasive, nonhazardous, contactless and inexpensive nature of acquiring information about the respiratory system using free field cough-sound registration makes it an attractive candidate for on-line follow-up and clinical diagnosis [10, 11]. However, due to the inability to extract adequate objective information and the lack of a full understanding of the origin of the cough sound, the application of this method is still limited [11]. The current auditory characterization of cough sounds leads to several common labels, such as brassing, barking, whooping, etc. $[11,12]$. It is imperative to obtain more quantitative and objective coughing-description by time-frequency analysis.

In view of this situation, the research presented by Hirtum and Berckmans [11] positively evaluates the vocal of the cough sound by estimating the global cough fundamental frequency or pitch. The objective is to evaluate the cough-sound origin towards vocal by assessing the fundamental frequency or pitch $[11,13]$.

In their study, the fundamental frequency was determined by autocorrelation analysis on both the original time-signal and the linear predicted time-signal. The experimental cough database was registered in the free acoustical field on respectively three pathological and nine healthy non-smoking human subjects and on two pathological and two healthy Belgian Landrace piglets. For both species differences between pitch values for cough-sounds originating from subjects suffering from a respiratory infection and healthy subjects were put forward. Finally, the retrieved pitch-difference between respectively healthy and infected subjects indicates the existence of acoustically different cough-classes in accordance with a different cause or physical condition of the respiratory system, which is illustrated in Fig. 1[11] and Table 1[11].

Table 1 Number (\#) and percentage (\%) of cough-sound recognized as voiced by autocorrelation analysis on respectively the rough time-signal and the LPC-reconstructed signal [11]

\begin{tabular}{lllll}
\hline & \multicolumn{3}{c}{ Rough } & \multicolumn{2}{c}{ LPC } \\
\cline { 2 - 5 } Cough & $\#$ & $\%$ & $\#$ & $\%$ \\
\hline Acute & 36 & 75 & 36 & 75 \\
Coluntary & 19 & 53 & 20 & 56 \\
Chronic & 1379 & 73 & 1379 & 73 \\
Chemical & 104 & 87 & 104 & 87 \\
\hline
\end{tabular}



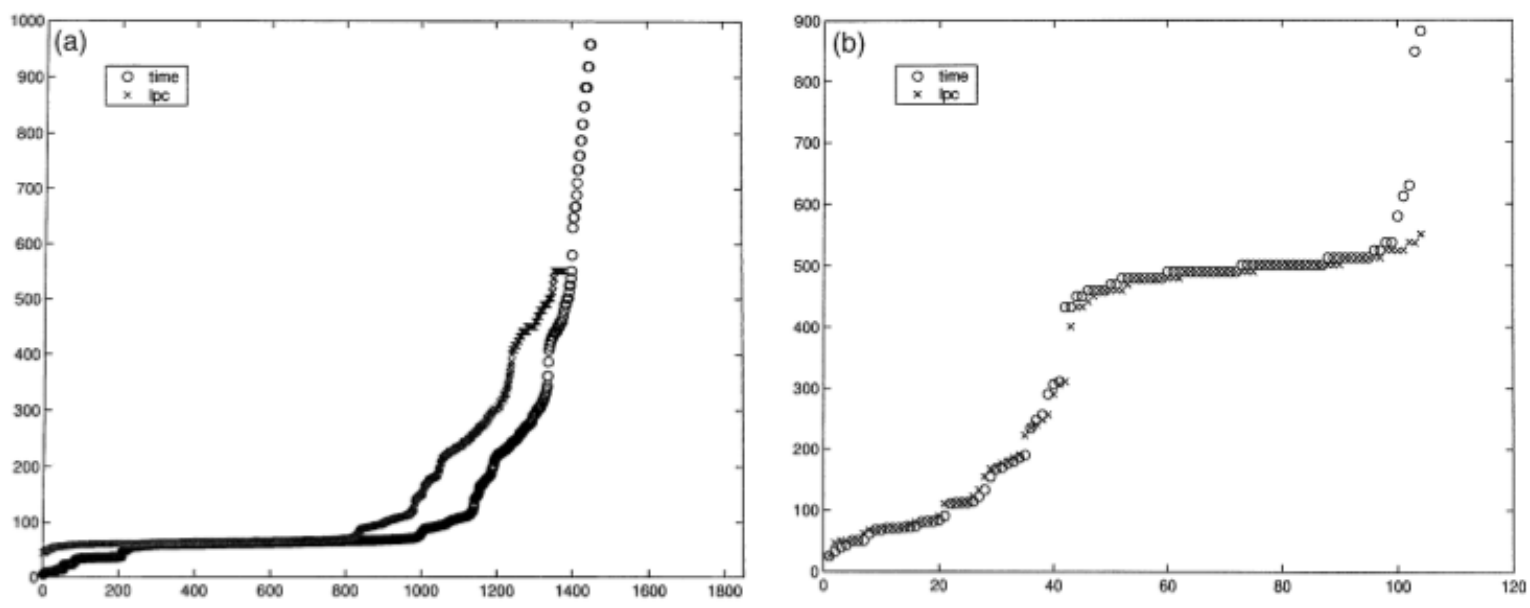

(c)
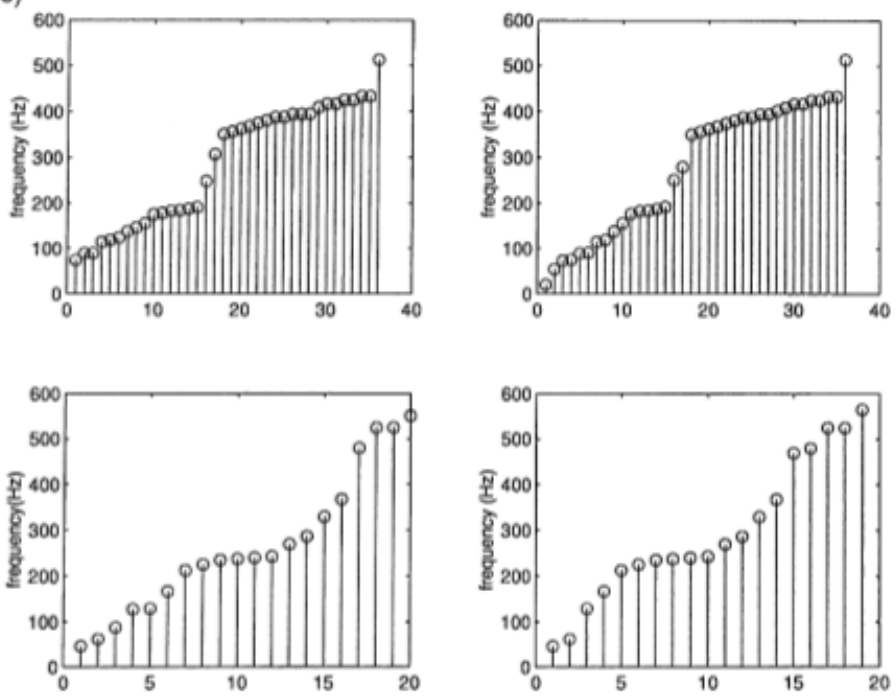

Fig.1 Estimated fundamental frequency for each cough-sound class-member in ascending order ([Hz]) for (top to bottom): 'chronic' (top 1), 'chemically’ (top 2), 'acute’ (top 3) and 'voluntary’ (top 4) cough sounds. The left plot in top 3 and top 4 is obtained on LPC predicted time-signals, the right column on original time-signals [11].

\section{Cough Sounds Discrimination}

As previously mentioned, coughing is a respiratory movement that occurs when the cough receptor is irritated physically or chemically. Information is transmitted to the central nervous system vagally and stimulates the cough center in the medulla oblongata portion of the brain stem [12]. The motor outputs are then sent through motoneurons to the respiratory muscles $[3,6]$. The analysis of cough may provide important clues not only to aid diagnosis, but also for the selection of drugs for treatment. As the cough in medicine is divided into a variety of types and a standard method to measure and analyze cough has not been established, this situation makes it difficult to diagnose the correct cough.

Akira Murata et al. [12] put forward that there are two types of coughs, productive and non-productive. The former is caused by excess airway secretions while the latter is due to chronic airway diseases. It is mentioned that both two types of coughs recorded in a free acoustic field were compared with those of voluntary cough of healthy subjects and were analyzed by sound spectrogram and time-expanded waveform. They concluded that all cough sounds could be separated into two or three phases which can be illustrated in Fig. 2[12] and Fig. 3[12]. 


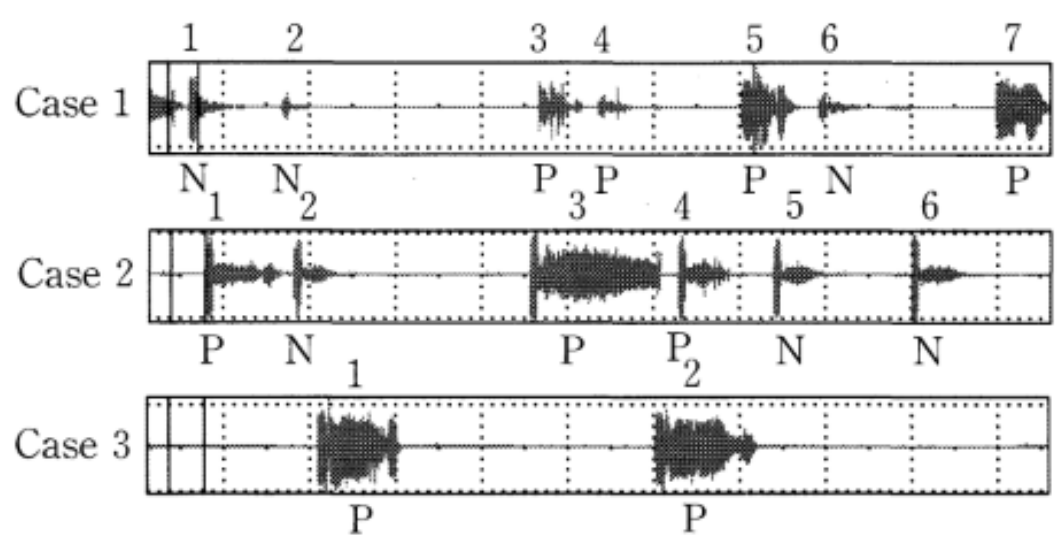

$\mathrm{N}$ : non-productive cough, $\mathrm{P}$ : productive cough

Fig.2 Examination of voluntary coughs

Voluntary coughs of patients with chronic bronchitis were examined. Productive coughs and non-productive coughs were both present in a series of voluntary coughs of three typical cases [12].

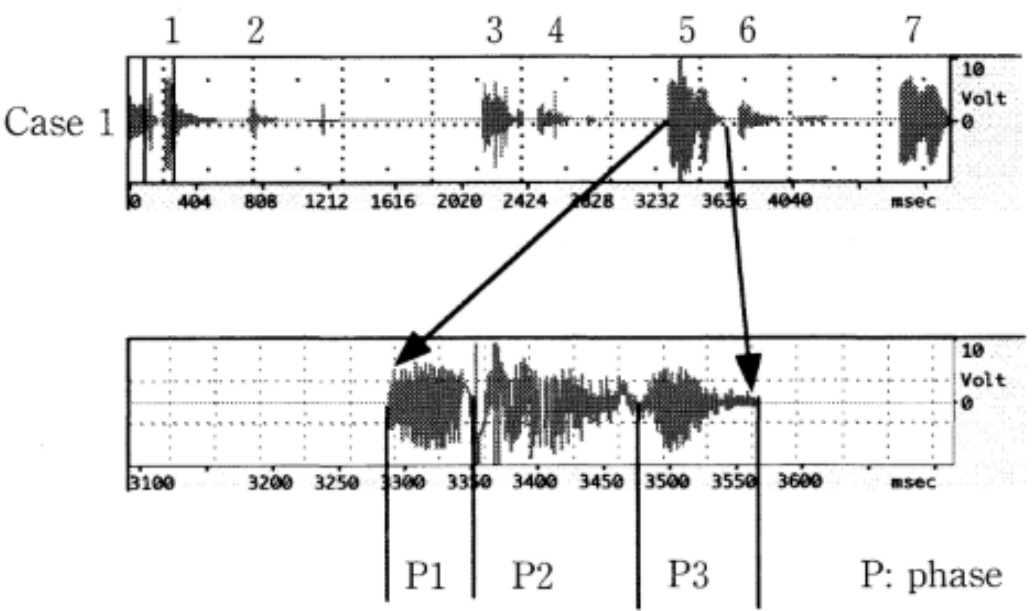

Fig.3 A time-expanded waveform of typical productive cough sounds

This figure shows fifth cough of case 1 from Fig. 2. The cough sounds could be separated into 2 or 3 phases. In analysis of one of the typical productive coughs of case 1 , three phases were identified. The duration of phase 1 was $50 \mathrm{msec}$, in phase $2105 \mathrm{msec}$, and in phase $390 \mathrm{msec}$. The total duration for all three phases was $245 \mathrm{msec}$. The wave patterns of sound pressure were distorted in phase 2 [12].

In addition, dry and wet cough types can be distinguished by the traditional cough sound discriminator algorithms. The performance of such algorithms, however, is affected by noise and reverberation in environment. Therefore, it is necessarily attempted to improve the performance of cough sound discriminator in reverberant environments.

Moradshahi and Chatrzarrin et al. [14] with the help of previously defined Linear Separation Score (LSS) [15] studied and quantitatively measured the effect of noise on the performance of cough sound discriminator. The authors found that a significant decrease in the performance of cough-sound discriminator in the presence of white noise using a single microphone for the acquisition of cough sound (Fig. 4 and Table 2) [14] while improvement in the performance of the cough-sound discriminator in the presence of white noise using microphone arrays (Fig. 5 and Table 3) [14].

Table 2 LSS of single microphone cough sound acquisition with white noise and variable cough sound volume [14]

\begin{tabular}{|c|c|c|c|c|c|c|c|}
\hline & \multicolumn{7}{|c|}{ Adobe Audition Volume Level (dB) } \\
\hline \multirow{2}{*}{ LSS } & $\mathbf{- 2 0}$ & $\mathbf{- 1 0}$ & $\mathbf{- 5}$ & $\mathbf{0}$ & $\mathbf{3}$ & $\mathbf{5}$ & $\mathbf{8}$ \\
\cline { 2 - 8 } & 0.61 & 0.69 & 0.69 & 0.71 & 0.71 & 0.73 & 0.69 \\
\hline
\end{tabular}




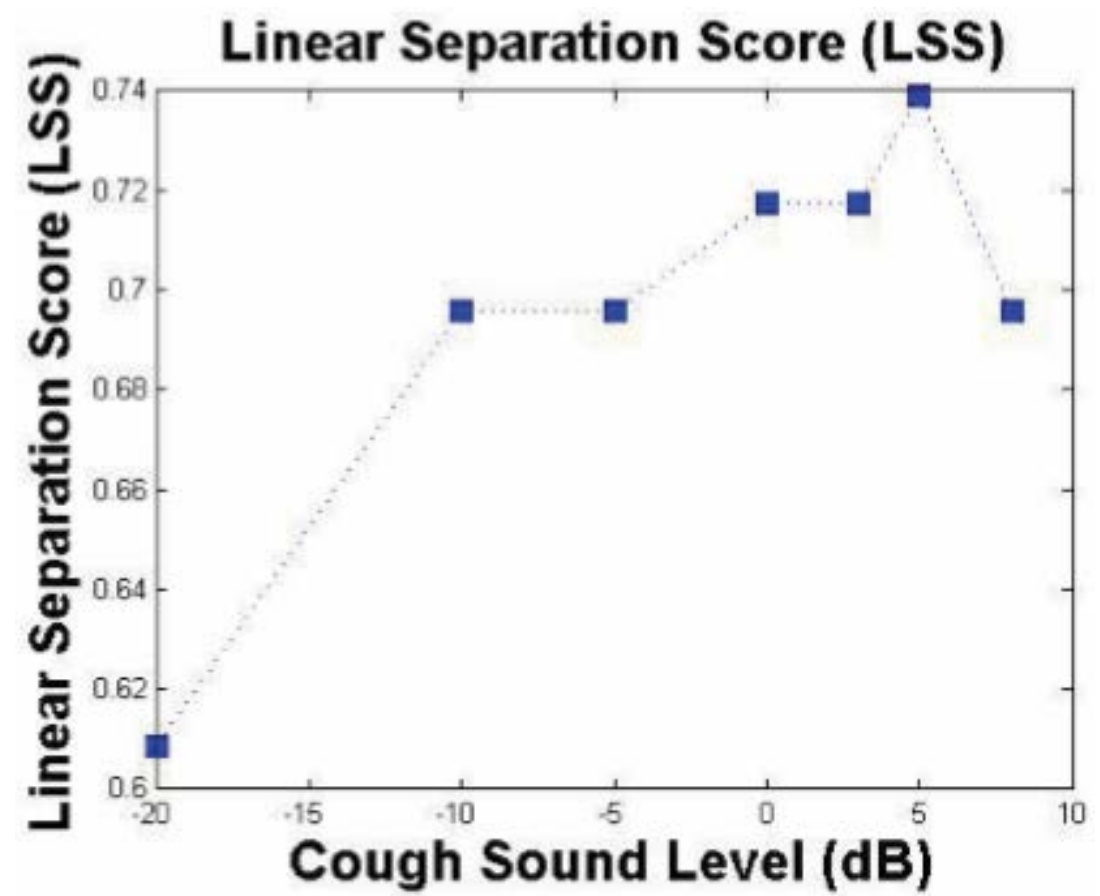

Fig.4 LSS of variable cough volume with single microphone [14]

Table 3 Microphone array LSS (DMic-Speaker = $50 \mathrm{~cm}$ ) [14]

\begin{tabular}{|c|c|c|c|c|}
\hline \multirow{2}{*}{ Num of Mics } & \multicolumn{4}{|c|}{ Microphone-to-Microphone Distance (cm) } \\
\cline { 2 - 5 } & $\mathbf{5}$ & $\mathbf{1 0}$ & $\mathbf{1 5}$ & $\mathbf{2 0}$ \\
\hline $\mathbf{1}$ & 0.81 & 0.83 & 0.81 & 0.81 \\
\hline $\mathbf{2}$ & 0.91 & 0.89 & 0.89 & 0.82 \\
\hline $\mathbf{3}$ & 0.93 & 0.95 & 0.91 & 0.82 \\
\hline $\mathbf{5}$ & 0.93 & 0.95 & 0.91 & 0.85 \\
\hline $\mathbf{7}$ & 0.95 & 0.95 & 0.93 & 0.89 \\
\hline
\end{tabular}

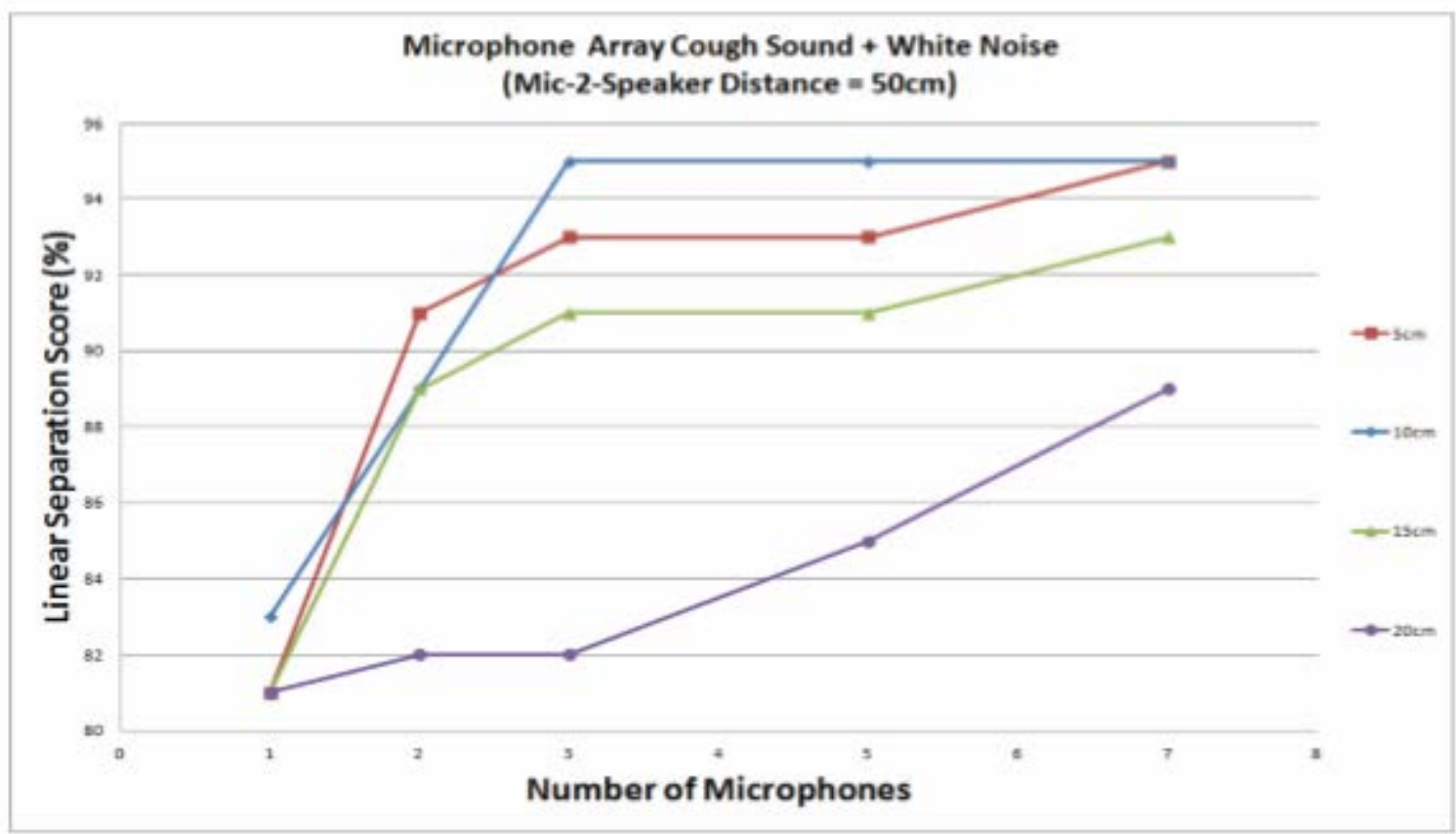

Fig.5 Microphone array LSS (DMic-Speaker = 50 cm) [14] 


\section{Application ideas}

The problem of attending to the health of people has become an important issue in developed countries. Through previous research, it is acknowledged that cough is closely related to human respiratory diseases. Therefore, it is a significant idea to put cough analysis into practical applications to benefit human's health.

Sung-Hwan Shin et al. [16] suggested that an automatic system that can monitor a health condition in real time using acoustical information and detect an abnormal symptom is applicable. The authors chose cough sound as a representative acoustical symptom of abnormal health conditions and proposed a hybrid model consisting of an artificial neural network (ANN) model and a hidden Markov model (HMM) to distinguish a cough sound from other environmental sounds. It is shown that the proposed hybrid model improved recognition rates on low SNR from $5 \mathrm{~dB}$ down to $-10 \mathrm{~dB}$ [16]. However, much time is required for the whole classification process. Therefore, for the development of this system, the problem of reducing the classification-time remains to be solved.

Amoh and Odame [17] put forward that a robust system for identifying and analyzing cough sounds is imperative as cough sounds can be very instrumental in the diagnosis of certain diseases and their intensity and frequency also infers the intensity of the particular illness. Then, the authors proposed wearable cough sensing methods to replace previous approaches which employ external environmental sensing methods to achieve detections [17]. However, the time required for the processing of analyzing cough data is still challenging and the pursuit of extreme processing speeds will lead to a huge system which will be very expensive and probably not applicable to wearable cough sensing methods. Besides, it is difficult to pack an equivalent processing power into a wearable continuous sensing device with current advances in microprocessors. Thus, for further work, the time required for the processing of analyzing cough data is a principal challenge and the advances in hardware like micro-processors also limit the development of this device.

\section{Conclusions}

Through the discussion above, it is concluded that the problems cough sound analysis confronted with are:

(1) The standard for cough collection has not been established yet.

(2) Reducing the time required for the processing of analyzing the cough data is demanded.

(3) The huge power required for continuous real-time processing is considerable challenging.

In view of the problems above, future work can proceed from the following aspects:

(1) Establish a unified standard shared cough database.

(2) A novel efficient algorithm for cough classification and processing is imperative.

(3) The development of micro-processor for the processing of cough analyzing is necessary.

As cough is closely related to people's health, the further study in this field is extremely vital to human.

\section{References}

[1] Banderali, G., Riva, E., Fiocchi, A., Cordaro, C. I. \& Giovannini, M. Efficacy and tolerability of levodropropizine and dropropizine in children with non-productive cough. Journal of International Medical Research 23, 175-183 (1995).

[2] Irwin, R. S., Rosen, M. J. \& Braman, S. S. Cough. A comprehensive review. Archives of Internal Medicine 137, 1186-1191 (1977).

[3] Brain, J. D., Proctor, D. F. \& Reid, L. Respiratory defense mechanisms. (Dekker\#, 1977).

[4] Corrao, W. M., Braman, S. S. \& Irwin, R. S. Chronic cough as the sole presenting manifestation of bronchial asthma. New England Journal of Medicine 300, 633-637 (1979).

[5] Hirschberg, J., Szende, T. \& Éliás, L. A clinical-acoustic study: Pathological cry, stridor and cough in infants. (Akadémiai Kiadó, 1982). 
[6] Korpas, J., Sadlonova, J., Salat, D. \& Masarova, E. The origin of cough sounds. Bulletin Europeen De Physiopathologie Respiratoire 23 Suppl 10, 47s-50s (1987).

[7] Korpás, J., Widdicombe, J. G. \& Vrabec, M. Influence of simulated mucus on cough sounds in cats. Respiratory Medicine 87, 49-54 (1993).

[8] Korpás, J., Widdicombe, J. G., Vrabec, M. \& Kudlicka, J. Effect of experimental lung oedema on cough sound creation. Respiratory Medicine 87, 55-59 (1993).

[9] Piirilä, P. \& Sovijärvi, A. R. Objective assessment of cough. European Respiratory Journal 8, 1949-1956 (1995).

[10] Korpás, J., Sadlonová, J. \& Vrabec, M. Analysis of the cough sound: an overview. Pulmonary Pharmacology 9, 261-268 (1996).

[11] Van, H. A. \& Berckmans, D. Assessing the sound of cough towards vocality. Medical Engineering \& Physics 24, 535 (2002).

[12] Murata, A. et al. Discrimination of productive and non-productive cough by sound analysis. Intern Med 37, 732-735 (1998).

[13] Rabiner, L. \& Juang, B. H. in Pearson Education, First Indian Reprint.

[14] Moradshahi, P., Chatrzarrin, H. \& Goubran, R. in Instrumentation and Measurement Technology Conference. 431-434.

[15] Moradshahi, P., Chatrzarrin, H. \& Goubran, R. in Instrumentation and Measurement Technology Conference. 20-23.

[16] Shin, S. H., Hashimoto, T. \& Hatano, S. Automatic detection system for cough sounds as a symptom of abnormal health condition. IEEE Transactions on Information Technology in Biomedicine A Publication of the IEEE Engineering in Medicine \& Biology Society 13, 486-493 (2009).

[17] Amoh, J. \& Odame, K. Technologies for developing ambulatory cough monitoring devices. Critical Reviews in Biomedical Engineering 41, 457 (2013). 